\title{
Circunferência da cintura, índice de massa corporal e fatores de risco cardiovascular na adolescência
}

\author{
Waist circumference, body mass index and cardiovascular \\ risk factors in adolescence
}

\author{
Rodrigo Bozza ${ }^{1}$ \\ Antonio Stabelini Neto ${ }^{1}$ \\ Anderson Zampier Ulbrich ${ }^{1}$ \\ Ítalo Quenni Araújo de Vasconcelos 1 \\ Luis Paulo Gomes Mascarenhas 1 \\ Lílian Messias Sampaio Brito ${ }^{1}$ \\ Wagner de Campos ${ }^{1}$
}

1 Universidade Federal do Paraná. Centro de Pesquisa em Exercício e Esporte. Curitiba, PR. Brassil

Recebido em 23/09/08 Reapresentado: 15/12/08 Aprovado: 13/02/09
Resumo - Embora diversos autores afirmem que a medida da circunferência da cintura (CC) é um indicador de fatores de risco cardiovascular mais confiável se comparado ao índice de massa corporal (IMC) e outros indicadores de adiposidade, estes resultados ainda não são conclusivos para população exclusivamente pediátrica. O Objetivo do presente estudo foi relacionar a $\mathrm{CC}$ e IMC com os fatores de risco para doenças cardiovasculares em adolescentes. A amostra foi composta por 108 rapazes e 133 moças (12 a 16 anos). A CC e o IMC foram mensurados seguindo procedimentos antropométricos usuais. Os fatores de risco para doenças cardiovasculares analisados foram a pressão arterial, colesterol total, LDL-c, HDL-c e triglicérides. Para as análises estatísticas, utilizaram-se estatística descritiva, testes de qui-quadrado e regressão logística, com $\mathrm{p}<0,05$. Os resultados sugerem que as moças com valores aumentados de IMC, apresentaram maior razão de chances de ter níveis indesejáveis de pressão arterial (OR:4,29; IC95\%:1,66-14,58). Em relação à CC, as moças com valores aumentados apresentaram maior razão de chances de apresentar pressão arterial elevada (OR:4,12;IC95\%:1,27-13,35) e colesterol total indesejável (OR:3,6;IC95\%:1,1-11,76). Apesar das poucas relações encontradas, principalmente para os rapazes, estes indicadores antropométricos podem ser úteis para a identificação e triagem de indivíduos com valores aumentados, possibilitando uma intervenção no sentido de incorporação de hábitos alimentares e de atividade física saudáveis, principalmente, em indivíduos jovens.

Palavras-chave: Dislipidemias; Hipertensão arterial, Circunferência da cintura; IMC.

Abstract - Although waist circumference (WC) has been suggested to be a more reliable cardiovascular risk factor than body mass index (BMI) and other indicators of adiposity, these results are not conclusive for an exclusively pediatric population. The objective of this study was to associate WC and BMI with cardiovascular risk factors in adolescents. The sample consisted of 108 boys and 133 girls (aged 12 to 16 years). WC and BMI were measured using standard anthropometric procedures. The cardiovascular risk factors analyzed were blood pressure, total cholesterol, LDL-c, HDL-c and triglycerides. Descriptive statistics, chi-square tests and logistic regression were used for statistical analysis $(p<0.05)$. Girls with increased BMI presented a higher odds ratio of undesirable blood pressure levels (OR: 4.29; 95\%CI: 1.66-14.58). The odds ratio of elevated blood pressure (OR: 4.12; 95\%CI: 1.27-13.35) and undesirable total cholesterol (OR: 3.6; 95\%CI:1.1-11.76) was higher in girls with increased WC. Despite the few associations observed, especially in boys, these anthropometric indicators might be useful for the identification and selection of individuals presenting elevated values, permitting intervention in terms of the incorporation of healthy dietary habits and physical activity, especially among young individuals. Key words: Dyslipidemia; Hypertension; Waist circumference; BMI. 


\section{INTRODUÇÃO}

Nos dias atuais, têm-se observado que mudanças no estilo de vida, como alterações nos hábitos alimentares e a inatividade física, contribuem para uma epidemia crescente de doenças crônicas degenerativas ${ }^{1}$. Entre elas, destaca-se a obesidade, que está associada diretamente com certos fatores de risco para doenças cardiovasculares como: hipertensão, baixos índices de HDL-c, altos níveis de colesterol total, LDL-c e triglicérides circulantes ${ }^{2,3}$.

Infelizmente, esse acréscimo na gordura corporal não é exclusividade da população adulta, pois dados recentes demonstram um aumento da prevalência de sobrepeso e obesidade também entre crianças e adolescentes ${ }^{4}$. Isto se torna preocupante, uma vez que manifestações na idade adulta resultam da interação entre uma variedade de fatores de risco que podem ter origem na idade pediátrica ${ }^{5}$.

Contudo, independentemente da quantidade de gordura corporal total, a distribuição da gordura no corpo pode ser um indicador mais preciso para a identificação dos fatores de risco cardiovasculares, tanto para adultos quanto para crianças ${ }^{4,6}$. Para indivíduos adultos, isto se confirma nos achados de Janssen et al. ${ }^{6}$, os quais observaram que sujeitos com valores de circunferência da cintura acima de $102 \mathrm{~cm}$ e $88 \mathrm{~cm}$ para homens e mulheres, respectivamente, mas com o IMC normal, apresentaram risco de doenças cardiovasculares semelhante ao risco de pessoas com sobrepeso e obesidade.

Neste sentido, embora diversos autores afirmem que a medida da circunferência da cintura é um indicador de fatores de risco cardiovascular mais confiável comparado ao IMC e a outros indicadores de adiposidade mais complexos ${ }^{6-8}$, estes resultados ainda não são conclusivos para população exclusivamente pediátrica. Por este motivo, o presente estudo teve como objetivo relacionar as classificações de circunferência da cintura e IMC propostos pela literatura com níveis de pressão arterial, colesterol total, lipoproteínas e triglicérides circulantes em adolescentes.

\section{PROCEDIMENTOS METODOLÓGICOS}

\section{Sujeitos}

A amostra não probabilística foi composta por 241 adolescentes, sendo 133 moças $(55,18 \%)$ e 108 rapazes $(44,81 \%)$, com idades entre 12 e 16 anos, matriculados na rede de ensino pública da cidade de São Mateus do Sul, PR. Esta amostra representa, aproximadamente, 9,71\% do total de alunos desta faixa etária matriculados no ano letivo de 2006 (total $=2.481$ alunos).

Os sujeitos do estudo foram recrutados nos dois colégios do município que tem maior representatividade da região urbana e rural, com 1.610 alunos, o que corresponde a $64,89 \%$ do total de alunos matriculados na rede pública de ensino do município. Foram convidados todos os alunos na faixa etária de 12 a 16 anos.

Os sujeitos que concordaram em participar do estudo receberam um "termo de consentimento" que foi preenchido pelos pais ou respectivos responsáveis, autorizando o uso dos seus dados.

\section{Instrumentos e procedimentos}

A circunferência da cintura (CC) foi mensurada no ponto médio entre o último arco costal e a crista ilíaca, utilizando-se uma fita antropométrica flexível, com escala de 0,1 centímetros?. A CC foi mensurada em duplicata e uma terceira medida foi realizada quando houve diferenças acima de $0,1 \mathrm{~cm}$ entre as medidas. A média das duas mensurações foi utilizada nas análises. Os sujeitos foram classificados conforme a proposta de Freedman et al. $^{10}$ : risco aumentado $\geq$ percentil 90 th. Na presente amostra, este percentil representou $77,2 \mathrm{~cm}$ para os rapazes e $71,44 \mathrm{~cm}$ para as moças.

A Massa corporal e estatura também foram mensuradas em duplicatas, sendo a média das duas medidas utilizada para calcular o IMC. Caso as medidas divergissem mais que $0,1 \mathrm{~kg}$ e $0,1 \mathrm{~cm}$ para a massa corporal e estatura, respectivamente, uma terceira medida era realizada. Para determinar a estatura total dos indivíduos foi utilizado um estadiômetro portátil, no qual o avaliado foi medido descalço, postado em posição anatômica sobre a base do estadiômetro, encostando a parte posterior do corpo e a cabeça posicionada no Plano de Frankfurt, estando em apnéia inspiratória no momento da medida?.

A massa corporal foi aferida com uma balança digital portátil, com resolução de $100 \mathrm{~g}$. O avaliado estava descalço, vestindo somente roupas leves, posicionado em pé e de costas para a escala da balança em posição anatômica, com a massa corporal igualmente distribuída entre ambos os pés 9 .

O índice de massa corporal (IMC) foi calculado através da divisão da massa corporal pelo quadrado da estatura9. A determinação do estado nutricional foi realizada através da utilização das tabelas de referência propostas por Conde e Monteiro ${ }^{11}$. Foram considerados com valores aumentados os indivíduos com sobrepeso e obesos. 
Tabela 1. Caracterização amostral dos adolescentes de ambos os sexos, com valores de média, desvio padrão, valores mínimos e máximos.

\begin{tabular}{lccc}
\hline & $\begin{array}{c}\text { Masculino } \\
\mathrm{n}=108\end{array}$ & $\begin{array}{c}\text { Feminino } \\
\mathrm{n}=133\end{array}$ & $\mathrm{t}$ \\
\hline $\mathrm{CC}(\mathrm{cm})$ & $69,3 \pm 6,7(56,5-91)$ & $64,97 \pm 5,7(50,5-89)$ & 5,333 \\
IMC $\left(\mathrm{Kg} / \mathrm{m}^{2}\right)$ & $20,09 \pm 2,7(14,6-29)$ & $19,87 \pm 2,9(12,5-31,3)$ & 0,0001 \\
PAS (mmHg) & $96,64 \pm 13,7(70-142)$ & $91,81 \pm 12,4(66-124)$ & 0,584 \\
PAD (mmHg) & $69,16 \pm 10,9(46-96)$ & $67,89 \pm 11,5(48-98)$ & 2,861 \\
CT (mg/dl) & $138,77 \pm 28,1(63-197)$ & $141,58 \pm 27,1(64-204)$ & 0,871 \\
HDL-c (mg/dl) & $44,54 \pm 9,9(29-75)$ & $47,18 \pm 14,3(20-86)$ & $-0,782$ \\
LDL-c (mg/dl) & $77,2 \pm 27,7(11-140)$ & $77,21 \pm 26,3(11-143)$ & $-1,74$ \\
TG (mg/dl) & $84,9 \pm 32,6(40-228)$ & $80,18 \pm 37,1(30-220)$ & $-0,005$ \\
\hline
\end{tabular}

CC - circunferência da cintura; IMC - índice de massa corporal; PAS - pressão arterial sistólica; PAD - pressão arterial diastólica; CT - colesterol total; TG - triglicérides.

A pressão arterial (PA) foi mensurada através do método auscultatório seguindo os parâmetros estabelecidos pelo The Fourth Report on the Diagnosis, Evaluation, and Treatment of High Blood Pressure in Children and Adolescents - NHBPEP'2. A pressão arterial sistólica (PAS) e diastólica (PAD) foram medidas no braço direito do avaliado com um esfignomanômetro de coluna de mercúrio, postado ao nível do coração e um estetoscópio (Pressão sistólica $=$ Korotkoff fase 1 e diastólica $=$ Korotkoff fase 5). A mensuração foi realizada após o indivíduo permanecer sentado em repouso por um período de 10 minutos. Duas leituras seguidas foram realizadas com intervalo de 10 minutos entre as medições, sendo considerado o valor médio entre as duas mensurações. Foi utilizado como ponto de corte para valores indesejáveis de hipertensão a PAS e/ou PAD $\geq$ percentil 90th (pré-hipertensos e hipertensos), de acordo com o sexo, idade e percentil da estatura ${ }^{12}$.

Foram coletados aproximadamente $8 \mathrm{ml}$ de sangue de cada indivíduo para análise laboratorial do perfil lipídico: colesterol total (CT), colesterol LDL (LDL-c), colesterol HDL (HDL-c) e triglicérides (TG). Na semana anterior a coleta, os sujeitos foram instruídos conforme as recomendações da Sociedade Brasileira de Cardiologia ${ }^{13}$ : a) jejum obrigatório de no mínimo $12 \mathrm{~h}$; b) não consumir bebidas alcoólicas na semana da coleta; c) evitar o abuso alimentar, em especial gordura, no dia anterior ao teste.

As amostras sanguíneas foram processadas e analisadas no mesmo dia da coleta. Para dosagem do CT mg/dl, HDL-c mg/dl e TG mg/dl foi utilizado o método enzimático-colorimétrico automatizado, através do aparelho da marca ABBOTT SPECTRUM, modelo CCX. O LDL-c mg/dl foi calculado pela fórmula de Friedewald et al..$^{14}$ (LDL-c $\left.=\mathrm{CT}-\mathrm{HDL}-\mathrm{c}-\mathrm{TG} / 5\right)$.

Os valores de referência para crianças e adolescentes sugeridos pela I Diretriz de Prevenção da Ate- rosclerose na Infância e Adolescência - $\mathrm{SBC}^{15}$ foram adotados para definir o perfil lipídico-lipoproteico de risco aterogênico. As dosagens consideradas como indesejáveis compreenderam os valores limítrofes e aumentados: CT>150mg/dl, LDL-c $>100 \mathrm{mg} / \mathrm{dl}$, HDL-c $\leq 45 \mathrm{mg} / \mathrm{dl}$ e TG $>100 \mathrm{mg} / \mathrm{dl}$.

Esta pesquisa foi aprovada pelo Comitê de Ética em pesquisa da UFPR (protocolo 018-06), conforme a resolução 196/96 do Conselho Nacional de Saúde que envolve pesquisas em seres humanos, em conformidade com a declaração de Helsinque de 1975.

\section{Análise estatística}

Para caracterização da amostra e apresentação dos dados, utilizou-se a estatística descritiva (média, desvio padrão, valores mínimos e máximos) e a comparação entre os sexos foi realizada por testes " $t$ " independentes. As variáveis categóricas (de acordo com os pontos de corte considerados) foram analisadas mediante tabelas de contingência, envolvendo testes de qui-quadrado $\left(\chi^{2}\right)$ para identificação de diferenças estatísticas nas prevalências apresentadas entre os sexos. A razão de chances dos indivíduos com valores aumentados de IMC e CC em apresentar os fatores de risco cardiovascular foi estabelecido mediante estimativas de Odds Ratio (OR), por intermédio da utilização da análise de regressão logística binária, adotando-se intervalo de confiança de 95\%, com nível alpha estipulado em $\mathrm{p}<0,05$ para todas as análises. As análises foram realizadas no software estatístico SPSS 13.0 (Chicago, IL).

\section{RESULTADOS}

Os valores encontrados para as variáveis através da análise descritiva são apresentados na tabela 1 .

$\mathrm{Na}$ tabela 2, são apresentadas às proporções de adolescentes que se encontram dentro dos valores 
Tabela 2. Prevalência de valores indesejáveis para IMC, pressão arterial e perfil lipídico nos adolescentes de ambos os sexos.

\begin{tabular}{lcccc}
\hline & \multicolumn{3}{c}{ Indesejáveis } \\
\cline { 2 - 5 } & Masculino & Feminino & $\chi^{2}$ & 0,232 \\
IMC & $17,6 \%$ & $12,1 \%$ & 1,428 & 0,79 \\
PA & $24,1 \%$ & $25,6 \%$ & 0,071 & 0,763 \\
CT & $35,2 \%$ & $33,3 \%$ & 0,091 & 0,091 \\
HDL-C & $60,2 \%{ }^{+}$ & $49,2 \%{ }^{+}$ & 2,865 & 0,57 \\
LDL-C & $22,2 \%$ & $19,2 \%$ & 0,323 & 0,009 \\
Triglicérides & $22,2 \%$ & $22,7 \%$ & 0,009 & 0 \\
\hline
\end{tabular}

IMC - índice de massa corporal; PA - pressão arterial; CT - colesterol total; *Diferença significativa $(p<0,05) ; \quad+$ sujeitos com valores de HDL-c $\leq 45 \mathrm{mg} / \mathrm{dL}$.

Tabela 3. Estimativas da razão de chances através do OR e IC entre o IMC e circunferência da cintura com os fatores de risco hemodinâmicos e bioquímicos.

\begin{tabular}{lcccc}
\hline & & IMC & & CC \\
\hline PA & Rapazes & Moças & Rapazes & Moças \\
CT & $2,14(0,7-6,2)$ & $4,29(1,66-14,58)$ & $2,34(0,6-9,1)$ & $4,12(1,27-13,35)$ \\
HDL-C & $0,4(0,13-1,45)$ & $2,19(0,76-6,31)$ & $0,83(0,2-3,42)$ & $3,6(1,1-11,76)$ \\
LDL-C & $2,12(0,7-6,42)$ & $0,79(0,27-2,26)$ & $1,58(0,38-6,51)$ & $0,87(0,27-2,75)$ \\
TG & $0,63(0,16-2,41)$ & $1,46(0,42-4,98)$ & $0,92(0,18-4,7)$ & $1,98(0,55-7,07)$ \\
\hline
\end{tabular}

IMC - índice de massa corporal; CC - circunferência da cintura; PA - pressão arterial; CT - colesterol total; TC - triglicérides

indesejáveis para os fatores de risco de doenças cardiovasculares, conforme os valores de referência propostos pela literatura para população pediátrica.

Embora a prevalência de sujeitos com níveis indesejáveis de HDL-c (Rapazes = 60,2\%; Moças $=49,2 \%$ ) tenha apresentado valores discrepantes entre os sexos, esta diferença não foi significativa em linguagem estatística. Da mesma forma, para o IMC, PA, CT, LDL-c e triglicérides, não houve diferenças significativas nas proporções entre moças e rapazes.

A razão de chances dos indivíduos com valores aumentados de CC e IMC em apresentar níveis indesejáveis de PA, CT, LDL-c, HDL e triglicérides circulantes é demonstrado na tabela 3, mediante estimativas de Odds Ratio (OR) e seus respectivos intervalos de confiança (IC).

Quanto ao índice de massa corporal, nota-se que, as moças com valores aumentados apresentam quase mais de 4 vezes mais razão de chances de apresentar níveis indesejáveis de pressão arterial. Para a circunferência da cintura, as moças com medidas aumentadas apresentaram em torno de 4 vezes maior razão de chances de apresentar a pressão arterial e o colesterol total elevados.

\section{DISCUSSÃO}

Embora diversos pesquisadores tenham direcionado seus estudos na elaboração de referenciais para me- didas de composição corporal como IMC e circunferência da cintura a partir de avaliação de amostras representativas da população pediátricic ${ }^{4,11,16}$, poucos estudos encontrados na literatura nacional avaliaram a relação da medida da circunferência da cintura e do IMC com fatores de risco para doenças cardiovasculares em crianças e adolescentes.

Ao analisar os resultados das prevalências apresentadas na tabela 2, observa-se que, em relação ao IMC a maioria dos sujeitos apresentou valores considerados normais para a idade e sexo, conforme as referências nacionais propostas por Conde e Monteiro ${ }^{11}$. Estes achados estão de acordo com estudos de prevalência realizados com amostras de adolescentes brasileiros ${ }^{5,17}$.

Equando se considera os fatores de risco hemodinâmicos e bioquímicos para doenças cardiovasculares avaliados nesta pesquisa, nota-se que a prevalência de sujeitos com valores indesejáveis para pressão arterial (rapazes $24,1 \%$ e moças $25,6 \%$ ) foi um pouco superior a encontrada por Guedes et al. ${ }^{5}$, embora este tenha avaliado separadamente as pressões sistólica e diastólica (PAD =10,2\% e 8,1\%; PAS= 8,8\% e 9,7\%, para rapazes e moças, respectivamente).

Em relação às prevalências encontradas por Gerber e Zielinsky ${ }^{17}$ (5\% para PAS e 3,2\% para PAD), a diferença encontrada foi mais evidente, pois estes autores consideraram somente valores acima do percentil 95th, enquanto que em nosso estudo consideramos valores acima do percentil 90th. 
Já em relação à avaliação do perfil lipídico, a proporção de indivíduos com valores indesejáveis de CT foi inferior às demonstradas por Giuliano et al..$^{18}(38 \%)$ e Seki et al. ${ }^{19}(46,6 \%)$, e superior ao relatado por Ribeiro et al. ${ }^{20}$ (32,9\%), Guedes et al. ${ }^{5}$ $(12,9 \%)$ e Gerber e Zielinsky ${ }^{17}$ (27,98\%). Entretanto, deve ser mencionado que diferentemente de nosso estudo, os valores considerados como indesejáveis por Guedes et al. ${ }^{5}$ foi $200 \mathrm{mg} / \mathrm{dl}$ e por Ribeiro et al. ${ }^{20}$ acima de $170 \mathrm{mg} / \mathrm{dl}$.

Para os sujeitos com valores diminuídos de lipoproteína de alta densidade (HDL-c), conforme os pontos de corte adotados pela Sociedade Brasileira de Cardiologia ${ }^{15}$,observou-se que a prevalência foi significativamente superior se comparada a outros estudos com amostras regionalizadas, que encontraram de $1,6 \%$ a 22,5\% de valores indesejáveis ${ }^{5,17-20}$. Neste caso, também foi encontrada diferença entre o valor de referência para o HDL-c e os utilizados por Guedes et al. ${ }^{5}(35 \mathrm{mg} / \mathrm{dl})$, e por Gerber e Zielinsky ${ }^{17}$ e Ribeiro et al. ${ }^{20}(40 \mathrm{mg} / \mathrm{dl})$.

Da mesma forma, houve discrepâncias nas proporções de indivíduos com valores indesejáveis de LDL-c, comparados a outros estudos ${ }^{5,17,18}$, além disso, igualmente ao CT e o HDL-c, os pontos de corte adotados em nosso estudo para valores indesejáveis foram diferentes aos propostos por outros autores.

Quanto aos triglicérides séricos, outros estudos ${ }^{18,19}$ encontraram uma prevalência de indivíduos com valores inadequados semelhante aos nossos dados (22\% e $22,5 \%$ respectivamente), porém, menores proporções foram encontradas em diferentes amostras nacionais ${ }^{5,17}$.

Analisando as associações dos valores de CC apresentados no presente estudo, $(77,2 \mathrm{~cm}$ para os rapazes e $71,44 \mathrm{~cm}$ para as moças.) com os fatores de risco para doenças cardiovasculares apresentadas na tabela 3, nota-se que este indicador esteve significativamente relacionado com a PA das meninas, confirmando os achados de Mikkola et al..$^{21}$ e Guimarães et al. ${ }^{22}$, embora estes autores não tenham realizado estas relações separadamente entre os sexos.

Além da PA, os valores aumentados da CC estiveram relacionados com valores indesejáveis de colesterol total. Neste sentido, Katzmarzyk et al. ${ }^{23}$ suportam a hipótese de que a CC pode ser usada efetivamente para a avaliação clínica da presença ou não de fatores de risco para doença cardiovascular em crianças e adolescentes. Esta afirmação é ratificada por Freedman et $\mathrm{al} .{ }^{24}$ que reconhecem a CC como preditora relevante de níveis alterados de lipídeos, lipoproteínas e apolipoproteinas em indivíduos jovens.

Quanto às associações dos valores aumentados de IMC, propostos por Conde e Monteiro ${ }^{11}$, com os fatores de risco hemodinâmicos para doenças cardiovasculares, relatos prévios demonstraram uma relação positiva dos níveis de pressão arterial com o IMC ${ }^{20,25}$. Da mesma forma, Mikkola et al. ${ }^{21}$ e Lusky et al. ${ }^{26}$ observaram que, quanto maior o valor de IMC maiores foram os valores de PAS e PAD, além disso, adolescentes que apresentam maior valor de IMC tem duas vezes mais chances de apresentar hipertensão arterial do que seus pares de IMC normal ${ }^{27}$. Esta relação do IMC elevado com a pressão arterial encontrada em outros estudos se confirma para as moças de nossa amostra, que demonstraram mais chances de apresentar hipertensão do que seus pares com peso normal.

Quanto às estimativas da razão de chances para indivíduos com IMC aumentado em relação ao perfil lipídico, observou-se neste estudo que não foram encontradas associações significativas. Estes resultados contrapõem outros estudos conduzidos com amostras nacionais e internacionais, nos quais foram encontradas associações significativas do IMC com as concentrações de glicose em jejum ${ }^{21}$, colesterol total ${ }^{20,28,29}$, LDL-c ${ }^{28,29}$, HDL-c ${ }^{28,29}$, triglicérides $^{28}$ e insulina ${ }^{29}$.

Uma das nossas limitações foi a comparação das prevalências de sujeitos com valores indesejáveis para o perfil lipídico com os estudos de outras regiões do Brasil, uma vez que diferentes critérios foram utilizados para classificação das dislipidemias, além de que, em alguns estudos, não foi realizada a coleta sanguínea em jejum prévio.

Através destes achados podemos concluir que apesar dos indicadores antropométricos analisados não terem se relacionado com os fatores de risco para doenças cardiovasculares nos rapazes, as moças com valores aumentados de IMC e CC apresentaram maior razão de chances de apresentar pressão arterial elevada e colesterol total elevado.

Apesar das poucas relações encontradas, principalmente para os rapazes, estes indicadores antropométricos podem ser úteis para a identificação e triagem de indivíduos com valores aumentados, possibilitando uma intervenção no sentido de incorporação de hábitos alimentares e de atividade física saudáveis, principalmente em indivíduos jovens.

Contudo, apesar do IMC ter uma referência nacional que possibilita a identificação de indivíduos com valores aumentados, a circunferência da cintura carece ainda de valores de referência nacionais, tendo-se que recorrer ao uso de percentis, impossibilitando a identificação da prevalência de sujeitos com valores aumentados. 


\section{REFERÊNCIAS BIBLIOGRÁFICAS}

1. Pitanga FJG. Epidemiologia, atividade física e saúde. Rev Bras Ciênc Mov 2002;10(3):49-54.

2. Kenchaiah S, Evans JC, Levy D, Wilson PWF, Benjamin EJ, Larson MG et al. Obesity and the risk of heart failure. N Engl J Med 2002;347(5):305-313.

3. Pozzan R, Pozzan R, Magalhães MEC, Brandão AA, Brandão AP. Dislipidemia, síndrome metabólica e risco cardiovascular. Rev SOCERJ 2004;17(2):97-104.

4. Fernandéz JR, Redden DT, Pietrobelli A, Allison DB. Waist circumference percentiles in nationally representative samples of African-American, EuropeanAmerican, and Mexican-American children and adolescents. J Pediatr 2004;145(4):439-444.

5. Guedes DP, Guedes JERP, Barbosa DS, Oliveira JA, Stanganelli LCR. Fatores de risco cardiovasculares em adolescentes: Indicadores biológicos e comportamentais. Arq Bras Cardiol 2006;86 (6):439-450.

6. Janssen I, Katzmarzyk PT, Ross R. Waist circumference and not body mass index explains obesity-related health risk. Am J Clin Nutr 2004(3);79:379-384.

7. Lean MEJ, Han TS, Seidell JC. Impairment of health and quality of life in people with large waist circumference. Lancet 1998;351(9106):853-856.

8. Taylor RW, Keil D, Gold EJ, Williams SM, Goulding A. Body mass index, waist girth and waist-to-hip ratio as indexes of total and regional adiposity in women: evaluation using receiver operating characteristic curves. Am J Clin Nutr 1998;67(1):44-49.

9. Tritschler K. Medida e avaliação em educação física e esportes de Barrow \& McGee. 5 ed. Barueri-SP: Manole, 2003.

10. Freedman DS, Serdula MK, Srinivasan SR, Berenson GS. Relation of circumferences and skinfold thicknesses to lipid and insulin concentrations in children and adolescents: the Bogalusa Heart Study. Am J Clin Nutr 1999;69(2):308-317.

11. Conde WL, Monteiro CA. Body mass index cutoff points evaluation of nutritional status in Brazilian children and adolescents. J. Pediatr 2006;82(4):266-272.

12. National High Blood Pressure Education Program Working Group on High Blood Pressure In Children And Adolescents. The Fourth Report on the Diagnosis, Evaluation, and Treatment of High Blood Pressure in Children and Adolescents. Pediatrics 2004;114(2):555-576.

13. Sociedade Brasileira de Cardiologia. Consenso brasileiro sobre dislipidemias: detecção, avaliação e tratamento. Arq Bras Cardiol 1996;67(supl):113-128.

14. Friedewald WT, Levy RI, Fredrickson DS. Estimation of the concentration of low-density lipoprotein cholesterol in plasma, without use of the preparative ultracentrifuge. Clin Chem 1972;18(6):499-502.

15. Sociedade Brasileira de Cardiologia. I Diretriz de prevenção da aterosclerose na infância e adolescência. Arq Bras Cardiol 2005;85(supl.6):1-36.

16. Kuczmarski RJ, Ogden CL, Grummer-Strawn LM, Flegal KM, Guo SS, Wei R et al. CDC growth charts: United States. Advance data from vital and health statistics; n.314. Hyattsville, Maryland: National Center for Health Statistics, 2000.

17. Gerber ZRS, Zielinsky P. Fatores de risco de aterosclerose na infância. Um estudo epidemiológico. Arq Bras Cardiol 1997;69(4):231-236.
18. Giuliano ICB Coutinho MSSA, Freitas SFT, Pires MMS, Zunino JN, Ribeiro RQC. Lipides séricos em crianças e adolescentes de Florianópolis, SC: Estudo Floripa Saudável. Arq Bras Cardiol 2005;85(2):85-91.

19. Seki M, Seki MO, Lima AD, Onishi MHO, Seki MO, Oliveira LAG. Estudo do perfil lipídico de crianças e jovens até 19 anos de idade. J Bras Patol Med Lab 2001;37(4):247-51.

20. Ribeiro RQC, Lotufo PA, Lamounier JA, Oliveira RG, Soares JF, Botter DA. Fatores adicionais de risco cardiovascular associados ao excesso de peso em crianças e adolescentes. O estudo do coração de Belo Horizonte. Arq Bras Cardiol 2006;86(6):408-418.

21. Mikkola I, Keinanen-Kiukaanniemi S, Laakso M, Jokelainen J, Harkonen P, Meyer-Rochowf VB et al. Metabolic syndrome in connection with BMI in young Finnish male adults. Diab Res Clin Pract 2007;76(3):404-409.

22. Guimarães ICB, Almeida AM, Santos AS, Barbosa DBV, Guimarães AC. Pressão arterial: efeito do índice de massa corporal e da circunferência abdominal em adolescentes. Arq Bras Cardiol 2008;90(5):426-432.

23. Katzmarzyk PT, Srinivasan SR, Chen W, Malina RM, Bouchard C, Berenson GS. Body Mass Index, Waist Circumference, and Clustering of Cardiovascular Disease Risk Factors in a Biracial Sample of Children and Adolescents. Pediatrics 2004;114(2):198-205.

24. Freedman DS, Khan LK, Dietz WH, Srinivasan SR, Berenson GS. Relationship of Childhood Obesity to Coronary Heart Disease Risk Factors in Adulthood: The Bogalusa Heart Study. Pediatrics 2001;108(3):712-718.

25. Garcia FD, Terra AF, Queiroz AM, Correia CA, Ramos PS, Ferreira QT et al. Avaliação de fatores de risco associados com elevação da pressão arterial em crianças. J Pediatr 2004;80:29-34.

26. Lusky A, Barell V, Lubin F, Kaplan G, Layani M, Shohat Z, et al. Relationship between Morbidity and Extreme Values of Body Mass Index in Adolescents Int J Epidemiol 1996;25(4):829-834.

27. Nielsen GA, Andersen LB. The association between high blood pressure, physical fitness, and body mass index in adolescents. Prev Med 2003;36(2):229-234.

28. Kim HM, Park J, Kim HS, Kim DH, Park SH. Obesity and cardiovascular risk factors in Korean children and adolescents aged 10-18 years from the Korean National Health and Nutrition Examination Survey, 1998 and 2001. Am J Epidemiol 2006;164(8):787-793.

29. Freedman DS, Dietz WH, Srinivasan SR, Berenson GS. The Relation of Overweight to Cardiovascular Risk Factors Among Children and Adolescents: The Bogalusa Heart Study. Pediatrics 1999;103(6):1175-1182.

\footnotetext{
Endereço para correspondência

Rodrigo Bozza

Universidade Federal do Paraná.

Departamento de Educação Física.

Rua Coração de Maria, 92. Jardim Botânico

Cep: 80215-370. Curitiba, PR. Brasil.

E-mail: rdbozza@gmail.com
} 